IJMMS 28:10 (2001) 621-623

PII. S0161171201006998

http://ijmms.hindawi.com

(c) Hindawi Publishing Corp.

\title{
ON THE EXTENSION OF LINEAR OPERATORS
}

\author{
JOHN J. SACCOMAN
}

(Received 7 March 2001)

\begin{abstract}
It is well known that the Hahn-Banach theorem, that is, the extension theorem for bounded linear functionals, is not true in general for bounded linear operators. A characterization of spaces for which it is true was published by Kakutani in 1940. We summarize Kakutani's work and we give an example which demonstrates that his characterization is not valid for two-dimensional spaces.
\end{abstract}

2000 Mathematics Subject Classification. 46A22.

1. Introduction. The fact that it is always possible to extend a bounded linear functional with its norm preserved was proven by Hahn in 1927 [3, page 217]. This is the well-known Hahn-Banach theorem, that is, the extension theorem for bounded linear functionals on normed linear spaces. The following theorem is Hahn's result with appropriate changes updating the terminology.

THEOREM 1.1. Let $M$ be a closed subspace of the real Banach space $X$, and $f a$ bounded linear functional defined on $M$. Then there exists a bounded linear functional $F$ defined on $X$ with $F(x)=f(x)$ for all $x \in M$ and $\|F\|=\|f\|$.

An obvious question is whether a similar result can be established for bounded linear operators. Specifically the question may be stated as follows.

QUESTION 1. Let $M$ be a closed subspace of the real Banach space $X$. Is it possible to extend every bounded linear operator $b$, which maps $M$ into an arbitrary Banach space $Y$, to a bounded linear operator $B$ which maps $X$ into $Y$ and has the same norm as $b$ ?

2. Kakutani's theorem. The above question was essentially raised by Kakutani [5, page 93]. He noted that the answer to this question in general is no. Actually, this was first demonstrated by Murray [6]. However, some results on extending linear operators have been obtained by placing conditions on the domain or range space. Papers by Bohnenblust [1], Kakutani [5], Murray [6], Nachbin [7], and Sobczyk [8] among others, contain such results.

Kakutani claimed that with some restrictions the answer to the above question is affirmative. These restrictions are given in the following theorem [5, page 94].

THEOREM 2.1 (see [5]). Let $X$ be a Banach space. In order that the answer to the above question be affirmative for any closed linear subspace $M$ of $X$ and any bounded linear 
operator which maps $M$ into another Banach space $Y$ (also arbitrary), it is necessary and sufficient that $X$ be a unitary space.

However, as it is shown in Section 3, there is still a problem with Kakutani's theorem.

3. A counterexample to Kakutani's theorem. The following definitions will aid in demonstrating the invalidity of Kakutani's claim.

DEFINITION 3.1. A normed linear space $X$ is a unitary space if the norm satisfies the parallelogram law, that is,

$$
\|x+v\|^{2}+\|x-v\|^{2}=2\left(\|x\|^{2}+\|v\|^{2}\right) \quad \forall x, v \in X .
$$

DEFINITION 3.2. Let $X$ be a Banach space and $M$ a closed subspace of $X$. Then $M$ has property $\mathscr{E}$ if for every Banach space $Y$ and every bounded linear operator $b$ mapping $M$ into $Y$, there exists a bounded linear operator $B$ mapping $X$ into $Y$ such that $B(x)=b(x)$ for all $x$ in $M$ and $\|B\|=\|b\|$.

DEFINITION 3.3. Let $X$ be a Banach space. We say that $X$ has the extension property, if every closed subspace of $X$ has property $\mathscr{E}$.

In other words, if $X$ has the extension property, then the answer to Question 1 is affirmative. In light of these definitions Kakutani's theorem may be restated as follows.

THEOREM 3.4. A Banach space $X$ has the extension property if and only if $X$ is a unitary space.

In 1935, Jordan and von Neuman [4] proved that the parallelogram law is a necessary and sufficient condition for a Banach space to be a Hilbert space. In fact Dunford and Schwartz in part 1 of their classic text "Linear Operators" use the Jordan-von Neuman condition to describe Kakutani's result [2, page 554].

Thus Kakutani proved (in 1939) that $X$ is a Hilbert space if and only if an arbitrary bounded linear operator defined on a subspace of $X$ has an extension to all of $X$ with the same norm.

In his proof of Theorem 2.1, however, Kakutani assumed that $\operatorname{dim}(X) \geq 3$. But he made no note of this restriction in the statement of his theorem and neither did Dunford and Schwartz. Of course, if every normed linear space $X$ with $\operatorname{dim}(X)<3$ is unitary, this would not be a problem. However, the vector space of pairs $\left(x_{1}, x_{2}\right)$ of real numbers with the norm defined by $\left\|\left(x_{1}, x_{2}\right)\right\|=\left|x_{1}\right|+\left|x_{2}\right|$ is not a unitary space. Furthermore, it can be shown that every two-dimensional Banach space has the extension property independent of whether it is a unitary space or not.

Let $X$ be any two-dimensional space, not necessarily a unitary space, $Y$ an arbitrary Banach space, and $M$ any subspace of $X$ ( $X$ is a Banach space and $M$ is closed since $X$ is finite dimensional). The following demonstrates that every subspace of $X$ has property $\mathscr{E}$.

The cases for two-dimensional and zero-dimensional subspaces are trivial. In the two-dimensional case set $B(x)=b(x)$, for all $x \in X$. In the zero-dimensional case set $B(x)=0$, for all $x \in X$. It is easy to see that in both cases $B$ is an extension of $b$ with $\|B\|=\|b\|$. 
Now let $M$ be any one-dimensional subspace of $X$. Then there is a $v$ in $X$ with $\|v\|=1$ and $M$ can be expressed as $M=\{t v \mid t$ is real $\|v\|=1\}$. If $b$ is any bounded linear operator mapping $M$ into $Y$, then

$$
\|b\|=\sup _{\|t v\| \leq 1}\|b(t v)\|=\|b(v)\| \sup _{|t| \leq 1}|t|=\|b(v)\| .
$$

The functional $f$ defined on $M$ by $f(t v)=t$ is linear and bounded with $\|f\|=1$. By Theorem 1.1, that is, the Hahn-Banach theorem, there is a bounded linear functional $F$ defined on $X$ with $F(x)=f(x)$ for all $x$ in $M$ and $\|F\|=\|f\|=1$. Let $B$ be the mapping from $X$ into $Y$ defined by $B(x)=F(x) b(v)$ with $x$ in $X$ and $v$ as defined previously, that is, $\|v\|=1$. Then $B$ is linear and

$$
B(t v)=F(t v) b(v)=t b(v)=b(t v)
$$

Further,

$$
\begin{aligned}
\|B\| & =\sup _{\|x\| \leq 1}\|B(x)\|=\sup _{\|x\| \leq 1}\|F(x) b(v)\| \\
& =\|b(v)\| \sup _{\|x\| \leq 1}|F(x)|=\|b(v)\|\|F\|=\|b(v)\|=\|b\| .
\end{aligned}
$$

Hence any one-dimensional subspace of $X$ has property $\mathscr{E}$.

Since every subspace of $X$ has property $\mathscr{E}, X$ has the extension property. But $X$ need not be a unitary space. In other words the correct form of Theorem 3.4 is as follows.

THEOREM 3.5. A Banach space $X$ with $\operatorname{dim}(X) \geq 3$ has the extension property if and only if $X$ is a unitary space, that is, if and only if $X$ is a Hilbert space.

\section{REFERENCES}

[1] F. Bohnenblust, Convex regions and projections Minkowski spaces, Ann. of Math. (2) 39 (1938), 301-308. Zbl 019.14101.

[2] N. Dunford and J. T. Schwartz, Linear Operators. I. General Theory, Pure and Applied Mathematics, vol. 7, Interscience, New York, 1958. MR 22\#8302. Zbl 084.10402.

[3] H. Hahn, Über lineare Gleichungssysteme in linearen Raümen, J. Reine Angew. Math. 157 (1927), 214-229 (German).

[4] P. Jordan and J. von Neumann, On inner products in linear metric spaces, Ann. of Math. 36 (1935), 719-723.

[5] S. Kakutani, Some characterizations of Euclidean space, Japan. J. Math. 16 (1939), 93-97. MR 1,146d. Zbl 0022.15001.

[6] F. J. Murray, On complementary manifolds and projections in spaces $L_{p}$ and $l_{p}$, Trans. Amer. Math. Soc. 41 (1937), no. 1, 138-152. CMP 1501 894. Zbl 016.21406.

[7] L. Nachbin, A theorem of the Hahn-Banach type for linear transformations, Trans. Amer. Math. Soc. 68 (1950), 28-46. MR 11,369a. Zbl 035.35402.

[8] A. Sobczyk, On the extension of linear transformations, Trans. Amer. Math. Soc. 55 (1944), 153-169. MR 5,272b. Zbl 063.07110.

John J. Saccoman: Department of Mathematics and Computer Science, Seton Hall UNIVERSITY, SOUTH ORANGE, NJ 07079, USA

E-mail address: saccomjj@shu.edu 


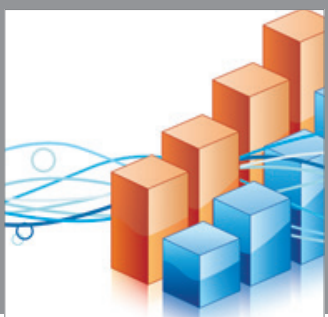

Advances in

Operations Research

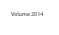

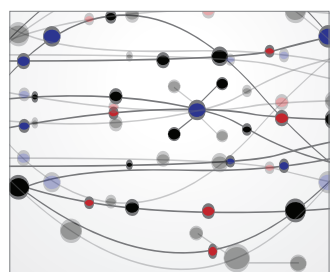

\section{The Scientific} World Journal
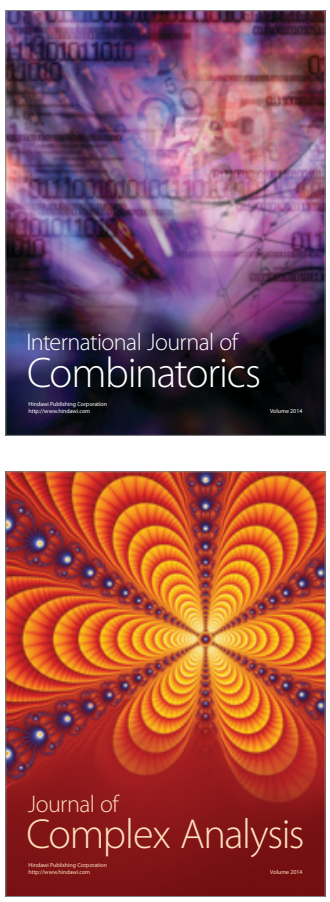

International Journal of

Mathematics and

Mathematical

Sciences
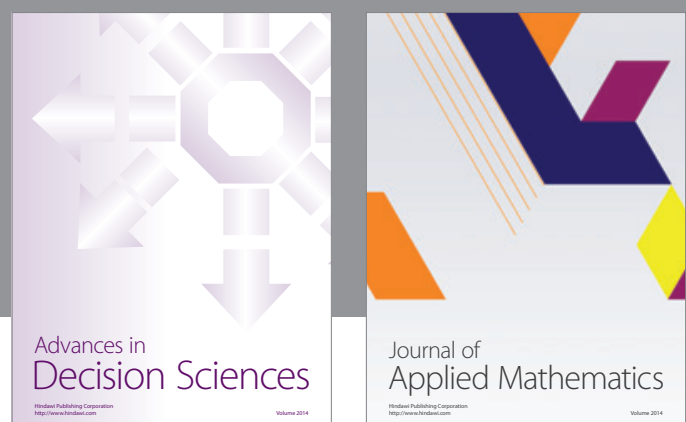

Journal of

Applied Mathematics
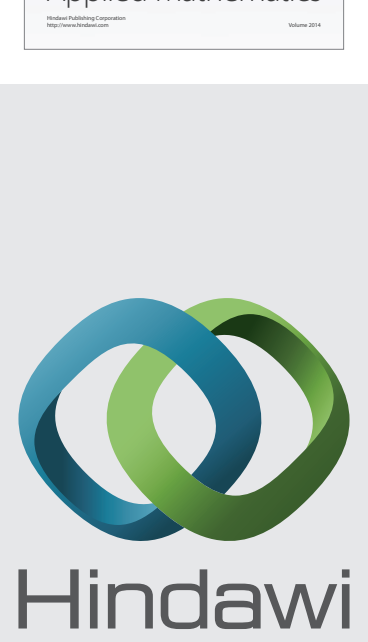

Submit your manuscripts at http://www.hindawi.com
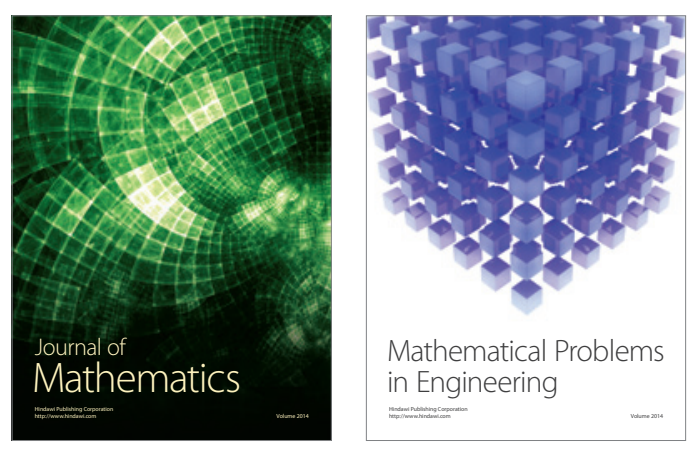

Mathematical Problems in Engineering
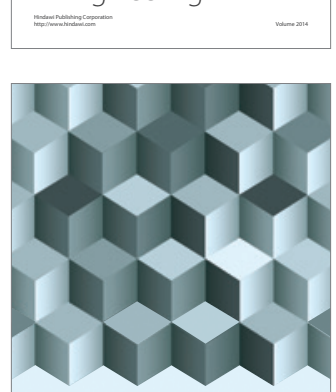

Journal of

Function Spaces
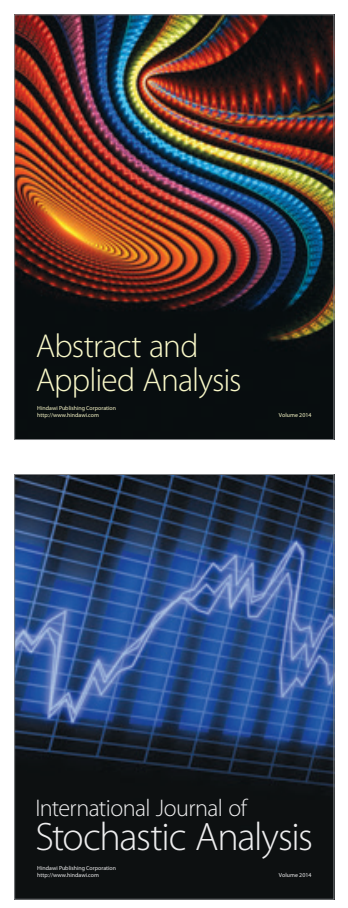

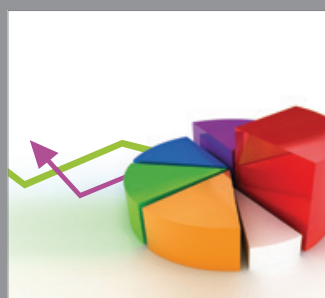

ournal of

Probability and Statistics

Promensencen
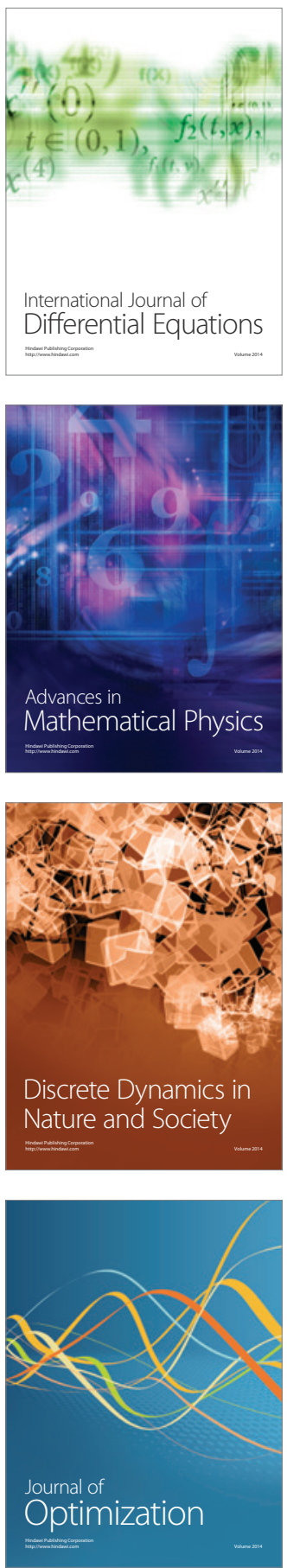